\title{
Screening of plant extracts and fractions on Aedes aegypti larvae found in the state of Mato Grosso do Sul (linnaeus, 1762) (culicidae)
}

\author{
KARLA R. DE ANDRADE PORTO ${ }^{1}$, PRISCILLA R. MOTTI ${ }^{1}$, MAMI YANO ${ }^{2}$, ANTONIA \\ R. ROEL ${ }^{1}$, CLAUDIA A.L. CARDOSO ${ }^{4}$ AND ROSEMARY MATIAS $^{3}$ \\ ${ }^{1}$ Universidade Católica Dom Bosco/UCDB, Av. Tamandaré, 6000, 79117-900 Campo Grande, MS, Brazil \\ ${ }^{2}$ Faculdade Estácio de Sá de Campo Grande, Mato Grosso do Sul, R.Venâncio Borges \\ do Nascimento, 377, 79050-700 Campo Grande, MS, Brazil \\ ${ }^{3}$ Universidade Estadual de Mato Grosso do Sul/UEMS, Rodovia Itahum, Km 12, 351, 79804-970 Dourados, MS, Brazil \\ ${ }^{4}$ Universidade Anhanguera - Uniderp, Av. Alexandre Herculano, 1400, 79037-280 Campo Grande, MS, Brazil
}

Manuscript received on January 9, 2015; accepted for publication on October 10, 2016

\begin{abstract}
The constant use of chemical insecticides for Aedes aegypti control has caused resistance in the mosquito populations. Thus, the objective of this study was to analyze the larvicidal potential of extracts and fractions of plants on $A$. aegypti larvae. The analysis included sixty one extracts and twenty five fractions of fifty botanical species at concentrations of $0.25 ; 0.12 ; 0.06$ to $0.03 \mathrm{mg} \mathrm{mL}^{-1} ; 4$ replications and one negative control of dechlorinate water and 1\% DMSO; and a positive control with rotenone. The toxicity index in descending order with $\mathrm{LC}_{50}$ for the most active of the extracts selected were ethanol extract of Ormosea arborea $\left(0.111 \mathrm{mg} \mathrm{mL}^{-1}\right)$ seeds and ethanol extracts of leaves such as Piper hispidum $\left(0.169 \mathrm{mg} \mathrm{mL}^{-1}\right)$, Solanum variabile $\left(0.188 \mathrm{mg} \mathrm{mL}^{-1}\right)$, O. arborea $\left(0.238 \mathrm{mg} \mathrm{mL}^{-1}\right)$, Turnera umifolia $\left(0.242 \mathrm{mg} \mathrm{mL}^{-1}\right)$ and Piper hispidum $\left(0.567 \mathrm{mg} \mathrm{mL}^{-1}\right)$. For plant fractions, the most active were chloroform $\left(0.192 \mathrm{mg} \mathrm{mL}^{-1}\right)$ and hexane $\left(0.342 \mathrm{mg} \mathrm{mL}^{-1}\right)$ P. aduncum leaves, hexane fraction $\left(0.415 \mathrm{mg} \mathrm{mL}^{-1}\right)$ and methanol extract $(0.625$ $\mathrm{mg} \mathrm{mL}^{-1}$ ) of Spermacocea latifolia leaves. Regarding the extract of T. umifolia single species, there is no bibliographic report on their degree of efficiency as an insecticide.
\end{abstract}

Key words: Dengue fever, insecticidal activity, insecticide from plant, larvicidal potential, disease vector.

\section{INTRODUCTION}

Brazil is considered an endemic area for dengue, which is transmitted by the mosquito Aedes aegypti (L.) (Culicidae) (Maciel et al. 2008). Dengue fever in Brazil, since 1986, has reached about 3 million cases. Only in the State of Mato Grosso do Sul, the year 2007 witnessed the largest outbreak of dengue

Correspondence to: Rosemary Matias

E-mail: rosematiasc@gmail.com fever $(69,378$ reports), with the city of Campo Grande recording the largest number of cases per capita ever reported in a state capital $(44,695$ cases), meaning one case per 16 residents (Oliveira et al. 2009).

Each year it is estimated that infections with dengue virus are responsible for more than 100 million of classic cases and more than 500 thousand cases of hemorrhagic dengue fever worldwide (Halstead 2007). In Brazil, from January to July 
$2010,942,153$ cases were reported in the country. In the same period of the previous year, 593,669 cases were reported, representing an increase of $58.7 \%$. The states with the highest incidence were Acre $(3,619.5$ cases per 100,000 inhabitants), Mato Grosso do Sul $(2,521.1$ cases per 100,000 inhabitants), Goiás (1,353.1 cases per 100,000 inhabitants), Rondônia (1,256.4 cases per 100,000 inhabitants), Roraima (1,146.9 cases per 100,000 inhabitants) and Mato Grosso (1,095.5 cases per 100,000 inhabitants). These six states make up $75 \%$ of cases in Brazil (Oliveira et al. 2011).

The safest way to combat or prevent a virus is immunization. However, other measures have been employed in the control and against vector spread, such as environmental sanitation, epidemiological surveillance, laboratory and research support, and education (Teixeira et al. 2009).

A current form of control is fogging insecticides such as organophosphate and pyrethroid, but their continuous use has resulted in resistance of populations (Guirado and Bicudo 2009) and exposure of operators (Vilela et al. 2010) to the compounds. These effects have been reported in Latin American countries, decreasing the effectiveness of this strategy in mosquito control (Rodríguez and Fernández 2007).

Chemical control with organophosphates proved to be inefficient in combating the mosquito. Among the organophosphates, temephos was reported as Aedes-resistant by Melo-Santos et al. (2010). Malathion and fenitrothion, which are used for controlling the adult stages of Aedes, are encountering resistance in different degrees (Pontes et al. 2005).

Concerns about the possible impacts on the environment and population health have allowed the development of products based on plants as an alternative to the use of synthetic chemicals (Oliveira Filho 2008). Studies have been developed to obtain products from plants with insecticidal properties (Furtado et al. 2005, Garcez et al. 2013).
In this study, we analyzed the insecticidal potential of plant extracts and fractions found in Mato Grosso do Sul on larvae of A. aegypti.

\section{MATERIALS AND METHODS}

SOURCE OF PLANT MATERIAL

Fifty plant species were collected from 1999 to 2012. Forty-eight came from different regions of the Cerrado and Pantanal in Mato Grosso do Sul and two exotic species from Campo Grande -MS (Melia azedarach L. e Nerium Oleander L.). The voucher specimens were sent to identification to the herbarium of Anhanguera - Uniderp University (by Eloty Justina Dias Schleder) and Federal University of Mato Grosso do Sul (by Vali Joana Pott and Arnildo Pott).

The plants subjected to the experiment in different extracts were: Anacardium humile St. Hil; Arachis hypogaea L.; Baccharis dracunculifolia DC.; Bambusa multiplex Lour. Raeusch.; Bambusa vulgaris var vittata Schrad. ex J.C. Wendl; Bauhinia fortificata Link.; Casearia silvestri Sw; Chenopodium ambrosioides L.; Cochlospermum regium Mart. ex Schrank Pilq.; Combretum leprosum Mart .; Crescentia cujete L.; Cymbopogon cittratus (D.C.) Stapf.; Diodia kuntzei K. Shum; Dimorphandra sp. Schott.; Equisetum pyramidale L.; Genipa americana L.; Guazuma ulmifolia Lamarck; Jacaranda cuspidifolia Marth.; Lafoensia pacari A. ST.-Hil.; Leucaena leucocephala (Lamark) de Wit; Maclura tinctoria (L.) D. Don ex Steud.; Melia azedarach L.; Malpighia glabras Linn; Myracrodruon urundeuva Allemão; Nerium oleander L.; Osmosea arborea Vell; Paullinia grandiflora St. Hill; Phoradendron sp. Nutt.; Piper aduncum L.; Piper amalago L.; Piper glabratum Kunt.; Piper hispidum Sw.; Piper vicosanum Yunk; Pouteria ramiflora (Mart.) Radlk; Randia armata Sw (DC); Richardia brasiliensis Gomes; Sebastiana hispida (Mart.) Pax; Solanum variabile Mart.; Spermacocea verticilata L.; Spermacocea 
grisebachii L.; Spermacocea latifolia L.; Stachytarpheta cayennensis Rich. Vahl; Sterculia apetala (Jacq.) H. Krast; Struthanthus flexicaulis M.; Tamarindus indicaL.; Tibouchina granulosa (Desr.) Cogn.; Tithonia diversifolia (Hemsl.) A. Gray; Turnera ulmifolia L.; Vernonia brasiliana L. (Druce); Vigna angularis Willd.

\section{PREPARATION OF EXTRACTS}

The plant material (leaves, stems, roots and fruits) were dried in an oven at $45{ }^{\circ} \mathrm{C}$. The extraction with different solvents occurred by continuous maceration until exhaustion of the plant extract, the solvent was then removed via rotary evaporator and concentrated to obtain a homogeneous emulsion. Part of the extracts were dissolved in methanol/ water and partitioned with solvents of different polarities (hexane, dichloromethane, chloroform and/or ethyl acetate).

The extracts and fractions of the species are presented in Table I. The descriptions are established in accordance with the updated nomenclature by the List of Species of the Brazilian Flora (2013).

\section{EXPERIMENTAL COLONY}

Collections of $A$. aegypti eggs were made in five neighborhoods in the city of Campo Grande, Mato Grosso do Sul, in partnership with the Center for Zoonosis Control (CCZ) using adapted methodology (Miyazaki et al. 2009, Gomes et al. 2007). The eggs were subjected to incandescent lighting for seven days until maturity. After that, F1 generation was produced and bioassays were conducted (Barata et al. 2001, Shaalan et al. 2005, Pontes et al. 2005).

The eggs were subjected to hatching in dechlorinate water and kept in Biochemical Oxygen Demand (BOD) incubator at $27 \pm 2^{\circ} \mathrm{C}$ and 14-hour photoperiod. The larvae were fed with cat food and water was daily replaced, as well as $\mathrm{pH}$ checked. In the adult stage, females were fed with blood supply every three days for 1.5 hours and males with $8 \%$ sugary food.

\section{BIOASSAYS}

Third stage larvae were selected for the testing, with larval density of $1: 1$ larvae/mL extract dissolved in dimethyl sulfoxide (DMSO) at $1 \%$. A negative control group of larvae was submitted to only dechlorinate water and DMSO at $1 \%$ and a positive control group to rotenone concentration from 0.002 to $0.020 \mathrm{mg} \mathrm{mL}^{-1}$ for 10,50 and $90 \%$ of the exposed population group. The analysis was performed in quadruplicate.

Samples of each extract were dissolved in $1 \%$ DMSO to obtain a solution of $0.5 \mathrm{mg} \mathrm{mL}^{-1}$. It was initially used for the preliminary tests with two replications per species for a toxicity general testing and then in dilutions of $0.25 ; 0.12 ; 0.06$ and $0.03 \mathrm{mg} \mathrm{mL}^{-1}$ in quadruplicate. The test results were checked after 24 hours of exposure.

\section{STATISTICAL ANALYSIS}

For the analysis of toxicity testing $\left(\mathrm{LC}_{10}, \mathrm{LC}_{50}\right.$ and $\mathrm{LC}_{90}$ ), parametric regression method Probit (Mclaughlin 1991) was performed using Leoraâ software (POLO 9735947870655352).

\section{RESULTS AND DISCUSSION}

The results of toxicity testing for larvae of $A$. aegypti at $0.5 \mathrm{mg} \mathrm{mL}^{-1}$ in 61 extracts, 25 fractions and 50 plant species are presented in Table I.

The extracts of S. grisebacei, B. dracunculifolia and $G$. americana showed larvicidal activity capable of causing death in $30 \%$ of the population at concentration of $0.5 \mathrm{mg} \mathrm{mL}^{-1}$. However, these extracts did not result in mortality at lower concentrations and hindered the calculation of $\mathrm{LC}_{50}$.

The extracts of $O$. arborea, T. ulmifolia and $S$. variabile, as well as extracts and fractions of $P$. aduncum L., P. hispidum L. and S. latifolia caused 
TABLE I

Activity of plant extracts on Aedes aegypti larvae at concentration of $0.5 \mathrm{mg} \mathrm{mL}^{-1}$.

\begin{tabular}{|c|c|c|c|c|}
\hline Species & Popular name & Plant organ & $\begin{array}{l}\text { Extract (Ext.)/ } \\
\text { Fraction (Fr.) }\end{array}$ & Death Percentage \\
\hline \multicolumn{5}{|c|}{ Amaranthaceae (1) } \\
\hline Chenopodium ambrosioides L. & $\begin{array}{l}\text { Erva de Santa } \\
\text { Maria }\end{array}$ & Leaves & Ethanolic ext. & - \\
\hline \multicolumn{5}{|c|}{ Anacardiaceae (2) } \\
\hline \multirow[t]{2}{*}{ Anacardium humile St. Hil } & \multirow[t]{2}{*}{$\begin{array}{l}\text { Cajuzinho do } \\
\text { Cerrado }\end{array}$} & \multirow[t]{2}{*}{ Leaves } & Ethanolic ext. & up to 30 \\
\hline & & & Hexane fr. & - \\
\hline \multirow{2}{*}{ Myracrodruon urundeuva Allemão } & \multirow{2}{*}{ Aroeira } & Leaves & \multirow{2}{*}{ Ethanolic ext. } & - \\
\hline & & Twig & & - \\
\hline \multicolumn{5}{|c|}{ Apocynaceae (3) } \\
\hline \multirow[t]{2}{*}{ Nerium Oleander L. } & Espirradeira & Leaves & Chloroform fr. & - \\
\hline & \multicolumn{3}{|c|}{ Asteraceae (4) } & \\
\hline \multirow[t]{2}{*}{ Baccharis dracunculifolia DC. } & Vassourinha & Leaves & Ethanolic ext. & up to 30 \\
\hline & & Leaves & Ethanolic ext. & - \\
\hline \multirow[t]{2}{*}{$\begin{array}{l}\text { Tithonia diversifolia (Hemsl.) A. } \\
\text { Gray }\end{array}$} & $\begin{array}{l}\text { Margaridão, } \\
\text { Girassol mexicano }\end{array}$ & & & \\
\hline & & Twig & Hexane fr. & - \\
\hline \multirow{2}{*}{$\begin{array}{l}\text { Vernonia brasiliana L. } \\
\text { (Druce) }\end{array}$} & \multirow{2}{*}{ Assa-peixe } & Leaves & \multirow{2}{*}{ Ethanolic ext. } & - \\
\hline & & Stems & & - \\
\hline \multicolumn{5}{|c|}{ Bignoniaceae (5) } \\
\hline Crescentia cujete L. & $\begin{array}{c}\text { Arvore de cuia, } \\
\text { cabaça }\end{array}$ & Leaves & Ethanolic ext. & - \\
\hline \multirow{3}{*}{ Jacaranda cuspidifolia Marth. } & & Leaves & \multirow{2}{*}{ Dichloromethane fr. } & - \\
\hline & Caroba & Stems & & - \\
\hline & & Leaves & Hexane fr. & - \\
\hline
\end{tabular}

Bixaceae (6)

\begin{tabular}{|c|c|c|c|c|}
\hline \multirow{7}{*}{$\begin{array}{c}\text { Cochlospermum regium Mart. ex } \\
\text { Schrank Pilq. }\end{array}$} & & Leaves & & - \\
\hline & & Twig & Ethanolic ext. & - \\
\hline & & Tubercle & & - \\
\hline & Algodão-do-campo & Leaves & Ethyl acetate fr. & - \\
\hline & & Leaves & & - \\
\hline & & Twig & Hexane fr. & - \\
\hline & & Tubercle & & - \\
\hline
\end{tabular}


TABLE I (continuation)

\begin{tabular}{|c|c|c|c|c|}
\hline Species & Popular name & Plant organ & $\begin{array}{l}\text { Extract (Ext.)/ } \\
\text { Fraction (Fr.) }\end{array}$ & Death Percentage \\
\hline \multicolumn{5}{|c|}{ Combretaceae (7) } \\
\hline Combretum leprosum Mart. & Carne-de-vaca & $\begin{array}{c}\text { Leaves } \\
\text { aerial parts }\end{array}$ & & - \\
\hline \multicolumn{5}{|c|}{ Equisetaceae (8) } \\
\hline \multirow{2}{*}{ Equisetum pyramidale L. } & \multirow{2}{*}{ Cavalinha } & \multirow{2}{*}{ aerial parts } & Ethanolic ext. & - \\
\hline & & & Methanolic ext. & - \\
\hline \multicolumn{5}{|c|}{ Euphorbiaceae (9) } \\
\hline \multirow{4}{*}{ Sebastiana híspida (Mart.) Pax } & \multirow{4}{*}{ Erva virgem } & \multirow{4}{*}{ Leaves } & Ethanolic ext. & - \\
\hline & & & Hexane fr. & - \\
\hline & & & Ethyl acetate fr. & - \\
\hline & & & $\begin{array}{c}\text { Methanolic fr. } \\
\text { hydro }\end{array}$ & - \\
\hline
\end{tabular}

Fabaceae (10)

\begin{tabular}{|c|c|c|c|c|}
\hline Arachis hypogaea L. & Amendoim & $\begin{array}{c}\text { Leaves } \\
\text { Twig }\end{array}$ & Ethanolic ext. & $\begin{array}{l}- \\
-\end{array}$ \\
\hline \multirow{2}{*}{ Bauhinia fortificata Link. } & Pata-de-vaca & Leaves & Ethanolic ext. & - \\
\hline & & & Ethanolic ext. & - \\
\hline \multirow[t]{2}{*}{ Dimorphandra sp. Schott. } & Faveiro & Fruits & Hexane fr. & - \\
\hline & & & Ethyl acetate fr. & - \\
\hline $\begin{array}{c}\text { Leucaena leucocephala (Lamark) } \\
\text { de Wit }\end{array}$ & Leucena & $\begin{array}{l}\text { Leaves } \\
\text { Twig }\end{array}$ & Ethanolic ext. & - \\
\hline \multirow{2}{*}{ Osmosea arborea Vell } & \multirow{2}{*}{ Dracena } & Leaves & \multirow{2}{*}{ Ethanolic ext. } & 100 \\
\hline & & Seeds & & 100 \\
\hline Tamarindus indica $\mathrm{L}$. & Tamarindo & Leaves & Ethanolic ext. & - \\
\hline \multirow[t]{2}{*}{ Vigna angularis Willd. } & Feijão azuki & Leaves & Ethanolic ext. & - \\
\hline & \multicolumn{3}{|c|}{ Flacourtiaceae (11) } & \\
\hline \multirow[t]{2}{*}{ Casearia silvestri $\mathrm{Sw}$} & Chá-de-frade & Leaves & Ethanolic ext. & - \\
\hline & \multicolumn{3}{|c|}{ Lythraceae (12) } & \\
\hline \multirow[t]{2}{*}{ Lafoensia pacari A. ST.-Hil. } & Mangabeira-brava & Leaves & Ethanolic ext. & - \\
\hline & Loran & eae (13) & & \\
\hline \multirow{2}{*}{ Struthanthus flexicaulis M. } & Erva de passarinho & Leaves & Ethanolic ext. & - \\
\hline & Mel & ae (14) & & \\
\hline \multirow[t]{2}{*}{ Melia azedarach $\mathrm{L}$. } & Cinamomo & Leaves & Ethanolic ext. & - \\
\hline & Malpi & ceae (15) & & \\
\hline Malpighia glabras Linn & Acerola & Leaves & Ethanolic ext. & - \\
\hline
\end{tabular}


TABLE I (continuation)

\begin{tabular}{|c|c|c|c|c|}
\hline Species & Popular name & Plant organ & $\begin{array}{l}\text { Extract (Ext.)/ } \\
\text { Fraction (Fr.) }\end{array}$ & Death Percentage \\
\hline \multicolumn{5}{|c|}{ Melastomataceae (16) } \\
\hline Tibouchina granulosa (Desr.) Cogn. & Quaresmeira & Leaves & Ethanolic ext. & - \\
\hline \multicolumn{5}{|c|}{ Moraceae (17) } \\
\hline $\begin{array}{c}\text { Maclura tinctoria (L.) D. Don ex } \\
\text { Steud. }\end{array}$ & $\begin{array}{l}\text { Amora brava, } \\
\text { taiuva }\end{array}$ & $\begin{array}{c}\text { Leaves } \\
\text { Twig }\end{array}$ & Ethanolic ext. & - \\
\hline \multicolumn{5}{|c|}{ Piperaceae (18) } \\
\hline \multirow{3}{*}{ Piper aduncum L. } & \multirow{3}{*}{ Pimenta de macaco } & \multirow{3}{*}{ Leaves } & Ethyl acetate fr. & 100 \\
\hline & & & Chloroform fr. & 100 \\
\hline & & & Hexane fr. & 100 \\
\hline Piper amalago L. & Jaborandi & Leaves & $\begin{array}{c}\text { Methanolic ext. } \\
\text { Hexane fr. }\end{array}$ & - \\
\hline Piper glabratum Kunt. & Piraparoba & Leaves & Ethanolic ext. & - \\
\hline \multirow{2}{*}{ Piper hispidum Sw. } & \multirow{2}{*}{ Jaborandi falso } & \multirow{2}{*}{ Leaves } & Methanolic ext. & 100 \\
\hline & & & Hexane fr. & 100 \\
\hline \multirow[t]{2}{*}{ Piper vicosanum Yunk } & Jaborandi & Leaves & Ethanolic ext. & - \\
\hline & \multicolumn{2}{|c|}{ Poaceae (19) } & & \\
\hline Bambusa multiplex Lour. Raeusch. & Bambu & Leaves & Ethanolic ext. & - \\
\hline $\begin{array}{l}\text { Bambusa vulgaris var vittata } \\
\text { Schrad. ex J. C. Wendl }\end{array}$ & Bambu & Leaves & Ethanolic ext. & - \\
\hline Cymbopogon cittratus (D.C.) Stapf. & Capim Citronela & Leaves & Ethanolic ext. & - \\
\hline \multicolumn{5}{|c|}{ Rubiaceae (20) } \\
\hline Diodia kuntzei K. Shum & $\begin{array}{l}\text { Planta aquática do } \\
\text { Pantanal }\end{array}$ & Leaves & $\begin{array}{l}\text { Methanolic ext. } \\
\text { Ethanolic ext. } \\
\text { Aqueous fr. }\end{array}$ & $\begin{array}{l}- \\
- \\
-\end{array}$ \\
\hline Genipa americana L. & Genipapo & $\begin{array}{l}\text { Leaves } \\
\text { Fruits }\end{array}$ & Ethanolic ext. & $\begin{array}{l}\text { up to } 30 \\
\text { up to } 30\end{array}$ \\
\hline Randia armata Sw (DC) & Fruta de cachorro, & Leaves & Methanolic ext. & - \\
\hline Richardia brasilienses Gomes & Erva-botão & Leaves & Ethanolic ext. & - \\
\hline \multirow[t]{2}{*}{ Spermacocea grisebachii L. } & \multirow[t]{2}{*}{ Agrião-do-campo } & \multirow[t]{2}{*}{ Leaves } & Methanolic ext. & up to 30 \\
\hline & & & Methanolic ext. & 100 \\
\hline Spermacocea latifolia $\mathrm{L}$. & Agriãozinho & Leaves & Hexane fr. & 100 \\
\hline Spermacocea verticilata $\mathrm{L}$. & $\begin{array}{l}\text { Vassourinha de } \\
\text { botão }\end{array}$ & Leaves & Ethanolic ext. & - \\
\hline \multicolumn{5}{|c|}{ Santalaceae (21) } \\
\hline \multirow{3}{*}{ Phoradendron sp. Nutt. } & \multirow{3}{*}{ Erva-de-passarinho } & Twig & Ethanolic ext. & - \\
\hline & & Twig & Aqueous fr. & - \\
\hline & & Leaves & Ethanolic ext. & - \\
\hline
\end{tabular}


TABLE I (continuation)

\begin{tabular}{|c|c|c|c|c|}
\hline Species & Popular name & Plant organ & $\begin{array}{l}\text { Extract (Ext.)/ } \\
\text { Fraction (Fr.) }\end{array}$ & Death Percentage \\
\hline \multicolumn{5}{|c|}{ Sapindaceae(22) } \\
\hline \multirow{3}{*}{ Paullinia grandiflora St. Hill } & & Leaves & \multirow{3}{*}{ Methanolic ext. } & - \\
\hline & Timbó & Twig & & - \\
\hline & & Seeds & & - \\
\hline \multicolumn{5}{|c|}{ Sapotaceae (23) } \\
\hline Pouteria ramiflora (Mart.) Radlk & Leitero preto, abiu & Leaves & Ethanolic ext. & - \\
\hline \multicolumn{5}{|c|}{ Solanaceae (24) } \\
\hline Solanum variabile Mart. & Jurubeba & Leaves & Ethanolic ext. & 100 \\
\hline \multicolumn{5}{|c|}{ Sterculiaceae (25) } \\
\hline & & Leaves & Ethanolic ext. & - \\
\hline Guazuma ulmifolia Lamarck & Chico magro & Twig & Ethanolic ext. & - \\
\hline & & Leaves & Aqueous fr. & - \\
\hline Sterculia apetala (Jacq.) H.Krast & $\begin{array}{c}\text { Mandovi, } \\
\text { Amendoim-de- } \\
\text { bugre }\end{array}$ & Leaves & Methanolic ext. & - \\
\hline \multicolumn{5}{|c|}{ Turneracea (26) } \\
\hline \multirow[t]{2}{*}{ Turnera ulmifolia $\mathrm{L}$. } & Erva damiana & Leaves & Ethanolic ext. & 100 \\
\hline & \multicolumn{4}{|c|}{ Verbenaceae (27) } \\
\hline \multirow{3}{*}{$\begin{array}{c}\text { Stachytarpheta cayennensis Rich. } \\
\text { Vahl }\end{array}$} & & Leaves & Ethanolic ext. & - \\
\hline & Gervão & Twig & Ethanolic ext. & - \\
\hline & & Leaves & Aqueous fr. & - \\
\hline
\end{tabular}

*30\%: percentage of minimum mortality for testing with toxicity indicative in plant extracts at different concentrations as established by McLaughlin (1991).

total mortality at a concentration of $0.5 \mathrm{mg} \mathrm{mL}^{-1}$ (Table I) and lethal concentrations (LC) at lower dilutions (Table II).

The ethanolic extract of $O$. arborea seeds $\left(\mathrm{LC}_{50} 0.111 \mathrm{mg} \mathrm{mL}^{-1}\right)$ was the most toxic showing high mortality at lower concentrations, followed by ethanolic extracts of $P$. hispidum $\left(0.169 \mathrm{mg} \mathrm{mL}^{-1}\right)$, S. variabile $\left(0.188 \mathrm{mg} \mathrm{mL}^{-1}\right)$, O. arborea $(0.238 \mathrm{mg}$ $\left.\mathrm{mL}^{-1}\right)$, T. umifolia $\left(0.242 \mathrm{mg} . \mathrm{mL}^{-1}\right)$ and $P$. hispidum $\left(0.567 \mathrm{mg} \mathrm{mL}^{-1}\right)$ leaves. The most active fractions were chloroform $\left(0.192 \mathrm{mg} \mathrm{mL}^{-1}\right)$ and hexane $\left(0.342 \mathrm{mg} \mathrm{mL}^{-1}\right)$ of $P$. aduncum leaves; and hexane fraction $\left(0.415 \mathrm{mg} \mathrm{mL}^{-1}\right)$ and methanol extract (0.625 $\left.\mathrm{mg} \mathrm{mL}^{-1}\right)$ of $S$. latifolia leaves (Table II).
Govindarajan (2009) reported that the Cassia fistula leaf extract (Fabaceae) holds larvicidal and ovicidal action for A. aegypti, as well as a repellent in the peridomestic environment. Similar studies with Casealpina ferrea (Fabaceae) seeds presented larvicidal activity with $85 \%$ efficiency for vectors of dengue and yellow fever (Cavalheiro et al. 2009).

Derivatives of the emulsion composed of different species of Copaifera showed insecticidal activity against vectors of $A$. aegypti and $A$. darlingi. The lethal concentrations for $A$. aegypti of Copaifera sp. $\mathrm{LC}_{50}$ showed values of $47 \mathrm{mg} \mathrm{L}^{-1}$ and $\mathrm{LC}_{90} 91 \mathrm{mg} \mathrm{L}^{-1}$. For C. guianensis, the values were 
TABLE II

Lethal Concentration (LC) in $\mathrm{mg} \mathrm{mL}^{-1}$ for the different extracts of Aedes aegypti larvae.

\begin{tabular}{|c|c|c|c|c|c|}
\hline Plant species & $\begin{array}{c}\text { Lethal } \\
\text { Concentration }\end{array}$ & $\begin{array}{c}\text { Average value in } \\
\text { correlation } 95 \%(\mathrm{mg} \\
\left.\mathrm{mL}^{-1}\right)\end{array}$ & \multicolumn{3}{|c|}{$\begin{array}{l}\text { Range limit in correlation } \\
\qquad 95 \% \\
\left(\mathrm{mg} \mathrm{mL}^{-1}\right)\end{array}$} \\
\hline \multirow{3}{*}{ Ormosea arborea Vell $^{\mathrm{A}}$} & $\mathrm{LC}_{10}$ & 0.164 & 0.133 & - & 0.185 \\
\hline & $\mathrm{LC}_{50}$ & 0.238 & 0.216 & - & 0.266 \\
\hline & $\mathrm{LC}_{90}$ & 0.347 & 0.307 & - & 0.429 \\
\hline \multirow{3}{*}{ Ormosea arborea $\mathrm{Vell}^{\mathrm{B}}$} & $\mathrm{LC}_{10}$ & 0.063 & 0.050 & - & 0.073 \\
\hline & $\mathrm{LC}_{50}$ & 0.111 & 0.098 & - & 0.124 \\
\hline & $\mathrm{LC}_{90}$ & 0.194 & 0.168 & - & 0.239 \\
\hline \multirow[t]{3}{*}{ Spermacoceae latifolia L. ${ }^{\mathrm{C}}$} & $\mathrm{LC}_{10}$ & 0.191 & 0.125 & - & 0.245 \\
\hline & $\mathrm{LC}_{50}$ & 0.415 & 0.341 & - & 0.512 \\
\hline & $\mathrm{LC}_{90}$ & 0.901 & 0.690 & - & 1.441 \\
\hline \multirow[t]{4}{*}{ Spermacoceae latifolia L. } & $\mathrm{LC}_{10}$ & 0.125 & 0.118 & - & 0.148 \\
\hline & $\mathrm{LC}_{50}$ & 0.625 & 0,618 & - & 0.653 \\
\hline & $\mathrm{LC}_{90}$ & 1.122 & 1.178 & - & 1.153 \\
\hline & $\mathrm{LC}_{10}$ & 0.097 & 0.062 & - & 0.124 \\
\hline \multirow[t]{3}{*}{ Solanum variabile Mart. ${ }^{\mathrm{A}}$} & $\mathrm{LC}_{50}$ & 0.188 & 0.153 & - & 0.232 \\
\hline & $\mathrm{LC}_{90}$ & 0.284 & 0.249 & - & 0.581 \\
\hline & $\mathrm{LC}_{10}$ & 0.122 & 0.118 & - & 0.190 \\
\hline \multirow[t]{2}{*}{ Piper aduncum L. ${ }^{\mathrm{C}}$} & $\mathrm{LC}_{50}$ & 0.342 & 0.206 & - & 0.401 \\
\hline & $\mathrm{LC}_{90}$ & 0.473 & 0.414 & - & 0.670 \\
\hline \multirow[t]{3}{*}{ Piper aduncum L. ${ }^{\mathrm{E}}$} & $\mathrm{LC}_{10}$ & 0.034 & 0.035 & - & 0.042 \\
\hline & $\mathrm{LC}_{50}$ & 0.192 & 0.188 & - & 0.195 \\
\hline & $\mathrm{LC}_{90}$ & 0.346 & 0.340 & - & 0.350 \\
\hline \multirow[t]{3}{*}{ Piper hispidum Sw. ${ }^{\mathrm{A}}$} & $\mathrm{LC}_{10}$ & 0.060 & 0.477 & - & 0.721 \\
\hline & $\mathrm{LC}_{50}$ & 0.169 & 0.169 & - & 0.189 \\
\hline & $\mathrm{LC}_{90}$ & 0.474 & 0.403 & - & 0.582 \\
\hline \multirow[t]{4}{*}{ Piper hispidum Sw. $^{\mathrm{E}}$} & $\mathrm{LC}_{10}$ & 0.320 & 0.264 & - & 0.366 \\
\hline & $\mathrm{LC}_{50}$ & 0.567 & 0.501 & - & 0.665 \\
\hline & $\mathrm{LC}_{90}$ & 1.003 & 0.818 & - & 1.410 \\
\hline & $\mathrm{LC}_{10}$ & 0.146 & 0.069 & - & 0.192 \\
\hline \multirow[t]{2}{*}{ Turnera ulmifolia Milsp ${ }^{\mathrm{A}}$} & $\mathrm{LC}_{50}$ & 0.242 & 0.182 & - & 0.334 \\
\hline & $\mathrm{LC}_{90}$ & 0.899 & 0.800 & - & 0.922 \\
\hline
\end{tabular}

$\mathrm{A}=$ Ethanolic extract of leaves. $\mathrm{B}=$ Ethanolic extract of seeds. $\mathrm{C}=$ Hexane extract of leaves. $\mathrm{D}=$ Methanolic extract of leaves. $\mathrm{E}=$ Chloroform extract of leaves.

$\mathrm{LC}_{50} 136 \mathrm{mg} \mathrm{L}^{-1}$ and $\mathrm{LC}_{90} 551 \mathrm{mg} \mathrm{L}^{-1}$ (Prophiro et al. 2012, Trindade et al. 2013).

The second major toxicity was obtained in ethanol extract of $P$. hispidum leaves. Piper has been described as effective insecticide. Studies with P. aduncum showed larvicidal activity of essential oil on A. aegypti (Oliveira et al. 2013).
The extracts of leaves and roots of $P$. aduncum and extracts of leaves, fruits and branches of $P$. tuberculatum at $0.5 \mathrm{~g} \mathrm{~L}^{-1}$ caused total mortality in A. aegypti larvae (Pohlit et al. 2004). The essential oils of $P$. gaudichaudianum, $P$. permucronatum, $P$. humaytanum and $P$. hostmanianum against $A$. aegypti larvae were analyzed. The most active oil was $P$. permucronatum $\mathrm{LC}_{50} 0.36 \mathrm{mg} \mathrm{mL}^{-1}$, 
followed by $P$. hostmanianum $\mathrm{LC}_{50} 0.54 \mathrm{mg} \mathrm{mL}^{-1}$ (Morais et al. 2007).

According to Misni et al. (2008), the essential oil of $P$. aduncum acts as an excellent repellent for A. aegypti. Subsequent studies showed that the essential oil at 8 and $10 \%$ led to significant mortality higher for $A$. aegypti (80\%) than for $A$. albopictus (71.6\%) (Misni et al. 2011).

The essential oil of $P$. marginatum inflorescences did not affect the oviposition when at concentration of $0.05 \mathrm{mg} \mathrm{mL}^{-1}$, but was effective in mortality of $A$. aegypti larvae at $\mathrm{LC}_{10}$ and $\mathrm{LC}_{50}$ of 0.0138 and $0.02 \mathrm{mg} \mathrm{L}^{-1}$, respectively (Autran et al. 2009). Sousa et al. (2008) reported low toxicity when analyzing the action of Piper species on $A$. aegypti larvae.

The grandisin lignan isolated from $P$. solmsianum caused larval mortality at $\mathrm{LC}_{50} 150 \mathrm{mg}$ $\mathrm{mL}^{-1}$. Histological analyzes reveal that the larvae had changes in the digestive tract, in the anterior and middle intestine with a tissue disorganization followed by death (Leite et al. 2012).

Other authors analyzed the essential oils of $P$. aduncum and P. hispidinervum on Tenebrio molitor (Fazolin et al. 2007), Piper aduncum and Piper hispodinervum on Sitophilus zeamais (Estrela et al. 2006), and P. aduncum on Cerotoma tingomarianus (Fazolin et al. 2005) reporting high toxicity. High insecticidal potential was attributed to $P$. tuberculatum extracts on larval stage of Spodoptera frugiperda (Castro 2007). Subsequently, Santos et al. (2010) describe the insecticidal activity of $P$. hispidum leaf extract and larvicidal potential on Hypothenemus hampei control.

According to the third $\mathrm{LC}_{50}$, the most toxic extract was obtained from $S$. variabile (Table I).

The aqueous extracts of Solanum villosum (Solanaceae) applied against Stegomyia aegypti (Culicidae) larvae caused mortality rates within 72 hours of exposure (Chowdhury et al. 2008).

A. aegypti larvae were subjected to Nicotiana tabacum (Solanaceae) extract. $\mathrm{LC}_{50}$ showed $0.45 \%$ and $0.12 \%$, and $\mathrm{LC}_{90} 0.98 \%$ and $0.25 \%$, respectively (Quirino 2010).

The larvicidal action on $A$. aegypti may be related to its antioxidant activity, causing inhibition of esterase, glutathione transferases or monooxygenases. This action was cited by Guirado et al. (2009) as one of the defense mechanisms of vectors and a common condition observed in resistant populations found in the national territory.

Preliminary phytochemical analysis of Capsicum frutescens var. longum showed high content of tannins, alkaloids, steroids and glycosides present in leaves and fruits (Vinayaka et al. 2010). They may be used as blockers of enzyme action since one of the mechanisms for larval mortality is related to the metabolism of esterases and other monooxygenase. Gupta et al. (2011) evaluated the larvicidal potential of seed $\alpha$-amylase inhibitor of Macrotyloma uniflorum in larvae, pupae and adults of $A$. aegypti and found adulticidal and larvicidal effect at a concentration of $0.2 \mathrm{mg} \mathrm{mL}^{-1}$.

To analyze the larvicidal potential of Cestrum nocturnum extract, Jawale et al. (2010) classified as active with $100 \%$ larvae mortality in 24 hours, setting $\mathrm{LC}_{100} 12 \mathrm{mg} \mathrm{L}^{-1}$ and $\mathrm{LC}_{50} 6 \mathrm{mg} \mathrm{L}^{-1}$. In accordance with the results obtained for the extracts of Plumbago zeylanica and C. nocturnum, the presence of bioactive phytochemicals influenced life cycle of $A$. aegypti by inhibiting the development of pupae and adult emergence (Patil et al. 2011).

The extract of T. umifolia leaves in this study was the only one with no reports of insecticidal activity. The genus Turnera (Turneraceae), known as chanana, has distribution in Brazil and South America. Santos et al. (2010) analyzed of the extract of T. ulmifolia leaves and observed the molluscicidal activity against Biomphalaria glabrata, and cytotoxicity with Artemia salina. The species has also been effective in controlling infectious forms of Trypanosoma cruzi and Leishmania (Santos et al. 2012). 
Reports from Nazar et al. (2009) and Dhanasekaran et al. (2013) describe the larvicidal and ovicidal potential and the repellent in ethanol extract of S. hispida against Anopheles stephensi, A. aegypti and Culex tritaeniorhynchus. The mortality of $A$. stephensi occurred at $\mathrm{LC}_{50} 89.45$ $\mathrm{mg} \mathrm{L}^{-1}$ and $100 \%$ repellency against female adult mosquitoes. Over $75 \%$ mortality was obtained from ethanolic extract of Spermacocea verticillata at concentration of $250 \mathrm{mg} \mathrm{L}^{-1}$ on the larvae of $A$. aegypti (Souza et al. 2013).

The data obtained in this study from larvicidal activity and literature information enabled the characterization of the species potential. The family(ies) evaluated proved its potential compared to the literature. However, it is recommended to continue the monitoring studies of sub-lethal dosages and fractionation of greater efficiency extracts for identification of main active principles which can be used as efficiency markers for other species.

\section{CONCLUSIONS}

This study shows the insecticide potential in plants found in Mato Grosso do Sul, Brazil. Forty-eight botanical species were analyzed and showed potential insecticide of $O$. arborea, S. latifolia, $S$. variabile, $P$. hispidum, $P$. aduncum and T. umifolia in order of efficiency for causing toxicity at lower concentrations.

\section{ACKNOWLEDGMENTS}

The Ministério de Ciência e Tecnologia (MCT), Conselho Nacional de Desenvolvimento Científico e Tecnológico (CNPq), Instituto Nacional de Ciência e Tecnologia em Áreas Úmidas (INAU), Centro de Pesquisa do Pantanal (CPP), Fundação de Apoio ao Desenvolvimento do Ensino, Ciência e Tecnologia do Estado de Mato Grosso do Sul (FUNDECT), Universidade Anhanguera - Uniderp and Universidade Católica Dom Bosco. The authors are grateful to the Conselho Nacional de Desenvolvimento Científico e Tecnológico (CNPq) for providing the research grant (PQ2).

\section{REFERENCES}

AUTRAN ES, NEVES IA, SILVA CSB, SANTOS GKN, CAMARA CAG AND NAVARRO DMAF. 2009. Chemical composition, oviposition deterrent and larvicidal activities against Aedes aegypti of essential oils from Piper marginatum Jacq. (Piperaceae). Bioresour Technol 100: 2284-2288.

BARATA EAMF, COSTA AIP, CHIARAVALLOTI FN, GLASSER CM, BARATA JMS AND NATAL D. 2001. População de Aedes aegypti (L.) em área endêmica de dengue, Sudeste do Brasil. Rev Saúde Publ 35: 237-242.

CASTRO MJP. 2007. Potencial inseticida de extratos de Piper tuberculatum JACQ. (Piperaceae) sobre a fase larval de Spodoptera frugiperda (J.E. Smith). Dissertação de mestrado, Mestrado em Agronomia, Universidade Federal do Piauí, 56 p. (Unpublished).

CAVALHEIRO MG, FARIAS DF, FERNANDES GS, NUNES EP, CAVALCANTI FS, VASCONCELOS IMV, MELO VMM AND CARVALHO AFU. 2009. Atividades biológicas e enzimáticas do extrato aquoso de sementes de Caesalpinia férrea Mart., Leguminosae. Braz J Pharmacog 19: 586-591.

CHOWDHURY N, GHOSH A AND CHANDRAG. 2008. Mosquito larvicidal activities of Solanum villosumberry extract against the dengue vector Stegomyia aegypti. BMC Complement Altern Med 8: 1-8.

DHANASEKARAN S, KRISHNAPPA K, ANANDAN A AND ELUMALAI K. 2013. Larvicidal, ovicidal and repellent activity of selected indigenous medicinal plants against malarial vector Anopheles stephensi (Liston.), dengue vector Aedes aegypti (Linn.) and Japanese encephalitis vector, Culex tritaeniorynchus (Giles.) (Diptera: Culicidae). J Agri Technol 9: 29-47.

ESTRELA JLV, FAZOLIN M, CATANI V, ALÉCIO MR AND LIMA MS. 2006. Toxicidade de óleos essenciais de Piper aduncum e Piper hispodinervum em Sitophilus zeamais. Pesq. Agropec Bras 41: 217-222.

FAZOLIN M, ESTRELA JLV, CATANI V, ALÉCIO MR AND LIMA MS. 2007. Propriedade inseticida dos óleos essenciais de Piper hispidinervum C. DC.: Piper aduncum L. e Tanaecium noctornum (Barb. Rodr.) Bur. \& K. Shun sobre Tenebrio molitor L. 1758. Ciênc Agrotecnica 31: 113-120.

FAZOLIN M, ESTRELA JLV, CATANI V, LIMA MS AND ALÉCIO MR. 2005. Toxicidade do óleo de Piper aduncum L. a adultos de Cerotoma tingomarianus Bechyné (Coleoptera: Chrysomelidae). Rev Neotrop Entomol 34: 485-489. 
FURTADO RF, LIMA MGA, ANDRADE NETO M, BEZERRA JNS AND SILVA MGV. 2005. Atividade larvicida de óleos essenciais contra Aedes aegypti L. (Diptera: Culicidae). Rev Neotrop Entomol 34: 843-847.

GARCEZ WS, GARCEZ FR, SILVA LMGE AND SARMENTO UC. 2013. Substâncias de Origem Vegetal com Atividade Larvicida Contra Aedes aegypti. Rev Virtual Quím 5: 363-393.

GOMES AC, SILVA NN, BERNAL RTI, LEANDRO AS, CAMARGO NJ, SILVA AM, FERREIRA AC, OGURA LC, OLIVEIRA SJ AND MOURA SM. 2007. Especificidade da armadilha Adultrap para capturar fêmeas de Aedes aegypti (Diptera: Culicidae). Rev Soc Bras Med Trop 40: 216-219.

GOVINDARAJAN M. 2009. Bioefficacy of Cassia fistula Linn. (Leguminosae) leaf extract against chikungunya vector, Aedes aegypti (Diptera: Culicidae). Eur Rev Med Pharmacol Sci 13: 99-103.

GUIRADO MM AND BICUDO HEMC. 2009. Alguns aspectos do controle populacional e da resistência a inseticidas em Aedes aegypti (Diptera, Culicidae). Bepa 6: 5-14.

GUPTA L, DESHPANDE S, TARE V AND SABHARWAL S. 2011. Larvicidal activity of the $\alpha$-amylase inhibitor from the seeds of Macrotyloma uniflorum (Leguminosae) against Aedes aegypt (Diptera: Culicidae). In J Tro Insect Sci 31: 69-74.

HALSTEAD SB. 2007. Dengue. Lancet 370: 1644-1652.

JAWALE C, KIRDAK R AND DAMA L. 2010. Larvicidal activity of Cestrum nocturnum on Aedes aegypti. Bangladesh J Pharmacol 5: 39-40.

LEITE ACCF, KATO MJ, SOARES ROA, GUIMARÃES AE, SANTOS-MALLET J AND CABRAL MMO. 2012. Grandisin caused morphological changes larval and toxicity on Aedes aegypti. Braz J Pharmacog 22: 517-521.

LISTA DE ESPÉCIES DA FLORA DO BRASIL. 2013. Disponivel: http://floradobrasil.jbrj.gov.br. Access: April 2013.

MACIEL IJ, SIQUEIRA JÚNIOR JB AND MARTELLI CMT. 2008. Epidemiologia e desafios no controle do dengue. Rev Patol Trop 37: 111-130.

MCLAUGHLIN, JL, CHANG C AND SMITH D.I. 1991. Bench-top bioassays for the discovery of bioactive natural products: an update. In: Atta-Ur-Rahman (Ed), Studies in Natural Products Chemistry. Amsterdam: Elsevier Science Publishers B.V 9, p. 383-409.

MELO-SANTOS MAV ET AL. 2010. Resistance to the organophosphate temephos: Mechanisms, evolution and reversion in an Aedes aegypti laboratory strain from Brazil. Acta Tropica 113: 180-189.

MISNI N, OTHMAN H AND SULAIMAN S. 2011. The effect of Piper aduncum Linn. (Family: Piperaceae) essential oil as aerosol spray against Aedes aegypti (L.) and Aedes albopictus Skuse. Trop Biomed 28: 249-258.

MISNI N, SULAIMAN NS AND OTHMAN H. 2008. The Repellent Activity of Piper aduncum Linn (Family: Piperaceae) Essential Oil against Aedes aegypti Using Human Volunteers. J Trop Med Parasitol 31: 63-69.

MIYAZAKI RD, RIBEIRO ALM, PIGNATTI MG, CAMPELO JUNIOR JH AND PIGNATI M. 2009. Monitoramento do mosquito Aedes aegypti (Linnaeus, 1762) (Diptera: Culicidae), por meio de ovitrampas no Campus da Universidade Federal de Mato Grosso, Cuiabá, Estado de Matogrosso. Rev Soc Bra Med Trop 42: 392397.

MORAIS SM, FACUNDO VA, BERTINI LM, CAVALCANTI ESB, ANJOS JÚNIOR JF, FERREIRA AS, BRITO ES AND SOUZA NETO MA. 2007. Chemical composition and larvicidal activity of essential oils from Piper species. Biochem Syst Ecol 35: 670-675.

NAZAR SR, WILLIAMS GP, ALI MS AND SUGANTHI P. 2009. Screening of Indian Coastal Plant Extracts for Larvicidal Activity of Culex quinquefasciatus. Indian J Chem Techn 2: 24-27.

OLIVEIRA ÉCL, PONTES ERJC, CUNHA RV, FRÓES ÍB AND NASCIMENTO D. 2009. Alterações hematológicas em pacientes com dengue. Rev Soc Bras Med Trop 42: 682-685.

OLIVEIRA FILHO EC. 2008. Avaliação da Periculosidade Ambiental de Bioinseticidas como uma Nova Perspectiva para a Ecotoxicologia no Brasil. J Braz Soc Ecotoxicol 3: $1-7$.

OLIVEIRA MSC, MORAIS SM, MAGALHÃES DV, BATISTA WP, VIEIRA EGP, CRAVEIRO AA, MANEZES JESA, CARVALHO AFU AND LIMA GPG. 2011. Antioxidant, larvicidal and antiacetylcholinesterase activities of cashew nut shell liquid constituents. Acta Trop 117: 165-170.

OLIVEIRA GL, CARDOSO SK, LARA JÚNIOR CR, VIEIRA TM, GUIMARÃES EF, FIGUEIREDO LS, MARTINS ER, MOREIRA DL AND KAPLAN MAC.2013. Chemical study and larvicidal activity against Aedes aegypti of essential oil of Piper aduncum L. (Piperaceae). An Acad Bras Cienc 85: 1227-1234.

PATIL CD, PATIL SV, SALUNKE BK AND SALUNKHE RB. 2011. Bioeficácia de Plumbago zeylanica (Plumbaginaceae) e Cestrum nocturnum (Solanaceae) extratos de plantas contra Aedes aegypti (Diptera: Culicide) e não-alvo de peixes Poecilia reticulata. Parasitol Res 108: 1253-1263.

POHLIT AM ET AL. 2004. Screening of plants found in the State of Amazonas, Brazil for larvicidal activity against Aedes aegypti larvae. Acta Amaz 34: 97-105.

PONTES RJS, REGAZZI ACF, LIMA JWO AND KERRPONTES LRS. 2005. Efeito residual de apresentações 
comerciais dos larvicidas temefos e Bacillus thuringiensis var. Israelensis sobre larvas de Aedes aegypti em recipientes com renovação de água. Rev Soc Bras Med Trop 38: 316-321.

PROPHIRO JS, SILVA MAN, KANIS LA, ROCHA LCBP, DUQUE-LUNA JE AND SILVA OS. 2012. First report on susceptibility of wild Aedes aegypti (Diptera: Culicidae) using Carapa guianensis (Meliaceae) and Copaifera sp. (Leguminosae). Parasitol Res 110: 699-705.

QUIRINO TF. 2010. Avaliação do potencial inseticida de solução de Nicotiana tabacum L. (Solanacea) para o controle de Aedes aegypti (L.) (Diptera:Culicidae). Trabalho de Conclusão de Curso (Graduação em Ciências Biológicas) - Universidade Estadual da Paraíba, Campina Grande, 54p. (Unpublished).

RODRÍGUEZ MM, BIEET JA AND FERNÁNDEZ D. 2007. Levels of insecticide resistance and resistance mechanisms in Aedes aegypti from some latin american countries. J Am Mosq Control Assoc 2: 420-429.

SANTOS AP, ZATTA DT, MORAES WF, BARA MTF, FERRI PH, SILVA MRR AND PAULA JR. 2010. Composição química, atividade antimicrobiana do óleo essencial e ocorrência de esteróides nas folhas de Pterodon emarginatus Vogel, Fabaceae. Braz J Pharmacog 20: 891896.

SANTOS KKA ET AL. 2012. Avaliação da atividade antiTrypanosoma e anti-Leishmania de Mentha arvensise Turnera ulmifolia. B Latinoam Caribe P1 11: 147-153.

SHAALAN EAS, CANYON DV, YOUNES MWF, WAHAB HA AND MANSOUR HA. 2005. Efects of sub - lethal concentrations of synthetic insecticides and Callitris glaucophylla extracts on the development of Aedes aegypti. J Vector Ecol 30: 295-298.
SANTOS VT, CANTO-DOROW TS AND EISINGER SM. 2010. Composição florística do componente herbáceo do Jardim Botânico da UFSM, Santa Maria, Rio Grande do Sul. Ciênc Nat 32: 61-82.

SOUSA PJC, BARROS CAL, ROCHA JCS, LIRA DS, MONTEIRO GM AND MAIA JGS. 2008. Avaliação toxicológica do óleo essencial de Piper aduncum L. Rev Bras Farmacog 18: 217-221.

SOUZA RKD, ALCANTARA MMAC AND PESSOA DA SILVA MA. 2013. Aspectos etnobotânicos, fitoquímicos e farmacológicos de espécies de Rubiaceaeno Brasil. Rev Cub Plant Med 18: 140-156.

TEIXEIRA MG, COSTA MC, BARRETO F AND BARRETO ML. 2009. Dengue: twenty-five years since reemergence in Brazil. Cad. Saúde Pública 25: S7-S18.

TRINDADE FTT, STABELI RG, PEREIRA AA, FACUNDO VA AND ALMEIDA E SILVA, A. 2013. Copaifera multijuga ethanolic extracts, oilresin, and its derivatives display larvicidal activity against Anopheles darling and Aedes aegypti (Diptera: Culicidae). Braz. J. Pharmacog 23: 464-470.

VILELA RAG, MALAGOLI ME AND MORRONE LC. 2010. Gerenciamento participativo em saúde do trabalhador uma experiência na atividade de controle de vetores. Saúde Soc 19: 969-980.

VINAYAKA KS, PRASHITH KEKUDA TR, NANDINI KC, RAKSHITHA MN, RAMYA M, SHRUTHI J, NAGASHREE GR AND ANITHA B. 2010. Potent insecticidal activity of fruits and leaves of Capsicum frutescens (L.) var. longa (Solanaceae). Pharm. Lett 2: 172-176. 